\title{
Compound lasing fiber optic ring resonators for sensing
}

\author{
S. Rota-Rodrigo*¹ ${ }^{1}$ M. González-Herráez ${ }^{2}$, M. Lopez-Amo ${ }^{1}$ \\ ${ }^{1}$ Departamento de Ingeniería Eléctrica y Electrónica, Universidad Pública de Navarra, Campus de Arrosadía, \\ 31006 Pamplona - Navarra, Spain. \\ ${ }^{2}$ Departamento de Electrónica, Universidad de Alcalá, Edificio Politécnico, Madrid 28871, Spain.
}

\begin{abstract}
In this work, we present a new method for increasing simultaneously the sensitivity and interrogation stability of fiberoptic intensity sensors. The configuration is based on a double-coupler ring resonator. On one side, the attenuation sensitivity of the sensor is enhanced by placing it inside a ring resonator. On the other side, the stability of the interrogation method is rendered close to perfect by creating an external lasing ring resonator with matched spectral response. The resulting lasers allow the stable interrogation of the sensors with an impressive sensitivity enhancement. In our experiments, the dynamic range of the intensity sensor (and consequently its sensitivity) is increased $15 \mathrm{~dB}$ by using this laser-based interrogation system. Simultaneously, the instability of the system is reduced to $\pm 0.05 \mathrm{~dB}$. In comparison with systems based on external interrogation, our method is $>100$ times more stable and also simpler, as no wavelength tunable elements are required.
\end{abstract}

Keywords: Optical fiber sensor, fiber laser sensor, laser ring resonator.

\section{INTRODUCTION}

Optical ring resonators (ORRs) have received increased attention in recent years, within the sensors field, due to their interesting optical properties, among them, the optical feedback, which provides strong attenuation sensitivity at the expense of a periodical response in frequency. The period of the ORR frequency response is inversely proportional to its length. In this regard, two main groups can be distinguished depending on the implementation technology; fiber optic ring resonators (FORRs) and integrated optic ring resonators (IORRs). IORRs have a high wavelength separation between resonances in comparison with FORRs, however due to their reduced size, they are aimed to chemical measures [1, 2], whereas FORRs allows measuring physical [3, 4]as well as chemical [5] parameters.

FORR is an interesting structure in combination with intensiometric sensors. In particular, FORRs allow a substantial increase in the sensitivity of the inserted sensors into the ring, due to the multiple recirculations of the light through the sensors. However it also leads to a considerable instability of the measurement. In systems based on amplitude detection, ultra-stable and ultra-narrow lasers are needed. In order to avoid this instability, a sweep interrogation with a tunable laser can be carried out [6] or, alternatively, an optical vector analyzer can be used. All these options render the interrogation system substantially more expensive. A lower cost alternative consists of using a time domain detection scheme, such as the cavity ring-down (CRD) technique [7] that analyzes the decay in light intensity when an input pulse is fed inside the cavity.

In this paper we present a specially designed fiber laser sensor systems based on FORRs which allows increasing the sensitivity in intensity sensor measurements while keeping good output intensity stability. The FORR is formed by a double coupler configuration, whose transmitted and reflected signals show complementary responses. The selfinterrogator laser was obtained by the combination of the FORR reflected signal with a gain medium. With this configuration the instability of the system is reduced in comparison to the use of external interrogators and also, the active equipment is reduced to an optical amplifier as gain medium and a semiconductor detector or a power meter as the detector.

*sergio.rota@unavarra.es; phone (0034) 948 169841; fax (0034) 948169720 


\section{EDFRL SENSOR SYSTEM CHARACTERIZATION}

The objective of this work is to obtain a laser-sensor system based on a resonant ring allowing an increase of both the sensitivity and the stability. Figure 1a shows the proposed ORR structure and its theoretical output power expressions, where, $E_{\mathrm{i}}$ is the input signal, $k_{\mathrm{i}}$ and $a_{\mathrm{i}}$, are the coupling ratios and the attenuations in the resonator, respectively, and $\mathrm{L}$ the total length of the ring. Figure $1 \mathrm{~b}$ and $1 \mathrm{c}$ show the comparison of the experimental and theoretical simulation results of the ring resonator in which is based our sensor, for a $k_{I}=k_{2}=0.9, L=2.14 \mathrm{~m}$ and $a_{1}=a_{2}=0.05 \mathrm{~dB}$ due to the splices between the couplers. As it is visible, the response of the ring is periodic in frequency, with resonant peaks spaced by a quantity $c / n L$ (around 95MHz). More importantly, the two outputs of the ORR show complementary responses.

(a)

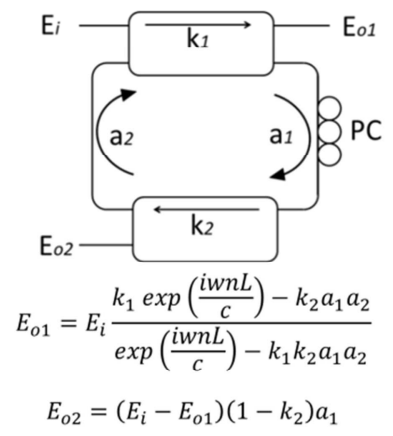

(b)

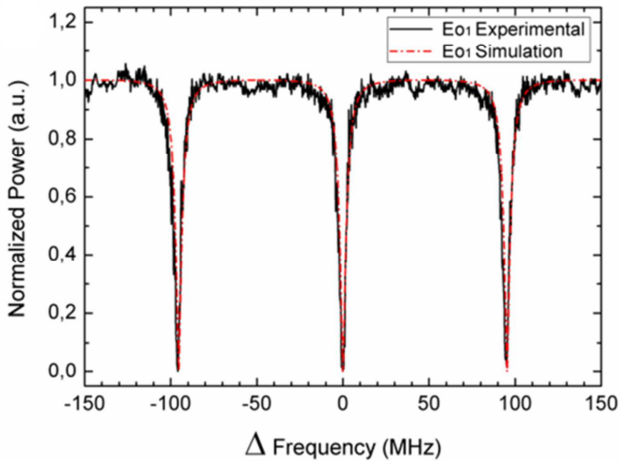

(c)

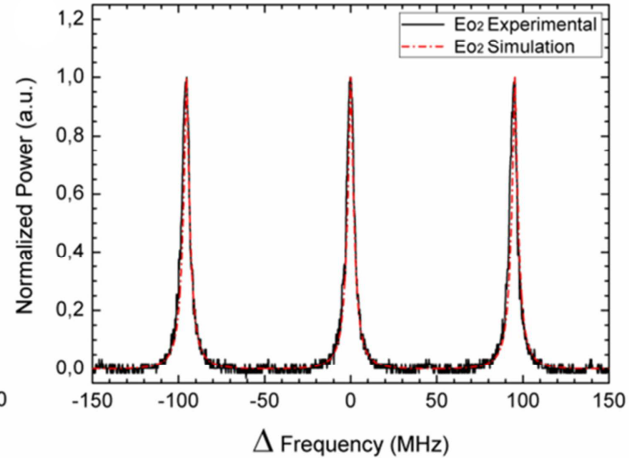

Fig. 1. (a) Fiber based ring resonator and , transmitted $\left(E_{\mathrm{o} 1}\right)$ and reflected $\left(E_{\mathrm{o} 2}\right)$ signal expressions of the FORR. (b) Experimental and simulated normalized spectra of the resonator for $E_{\mathrm{o} 1}$, and (c) $E_{\mathrm{o} 2}$.

The proposed system uses the resonant output $E_{\mathrm{o} 2}$ of the ORR as seed for generating a fiber ring laser. This way the structure itself behaves as the interrogator of the sensor. The configuration is shown in figure 2 where, the gain medium was an erbium doped fiber amplifier (EDFA: EFA-P21 from MPB Technologies), a filter at $1533.9 \mathrm{~nm}$ with a BW of 0.1 $\mathrm{nm}$ was used for the laser wavelength selection and a power meter (Ando Aq2140) as detector. Note that the lasing cavity in figure 2 is much longer than the ORR cavity, so effectively the spectral response of the obtained laser turns out to be approximately that of $E_{02}$.

The characterization was carried out using a variable attenuator (VA), simulating an intensity sensor element. For this purpose a voltage controlled VA was used in order to increase the precision and the resolution of the characterization. As can be shown in figure 2, there are two positions A and B, where the VA can be located. Placing alternatively the VA in these two positions we can compare the behavior of a sensor inside the ring (B) and outside it (A). Furthermore, by disconnecting the internal ORR (dash line in figure 2) the sensor can be compared with a simple ring configuration. A series of measurements varying the applied voltage of the VA from 0 to $4 \mathrm{~V}$ with steps of $10 \mathrm{mV}$ was carried out.

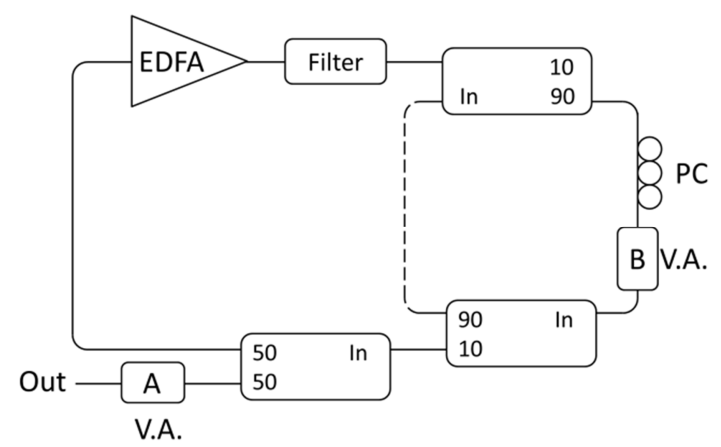

Fig. 2. Experimental setup of the EDFRL sensor system.

As can be seen in figure 3a, when the VA was located inside the ring, the dynamic range of the system can be increased up to $15 \mathrm{~dB}$. The attenuation operation range was defined from $0 \mathrm{~dB}(0 \mathrm{~V})$ to $7.5 \mathrm{~dB}(3.25 \mathrm{~V})$, where the loss of the 
cavity finally prevents the laser condition. Also the proposed system shows strong improvements in sensitivity compared to the open-loop response of the sensor and also in comparison with the conventional lasing ring resonator.

In order to determinate the system instability, a study of the output power variations following the VA attenuation inside the ring was performed. The system was tested for 11 different voltage values of the VA and each measurement was carried out along 30 minutes, using an acquisition time of 10 seconds.
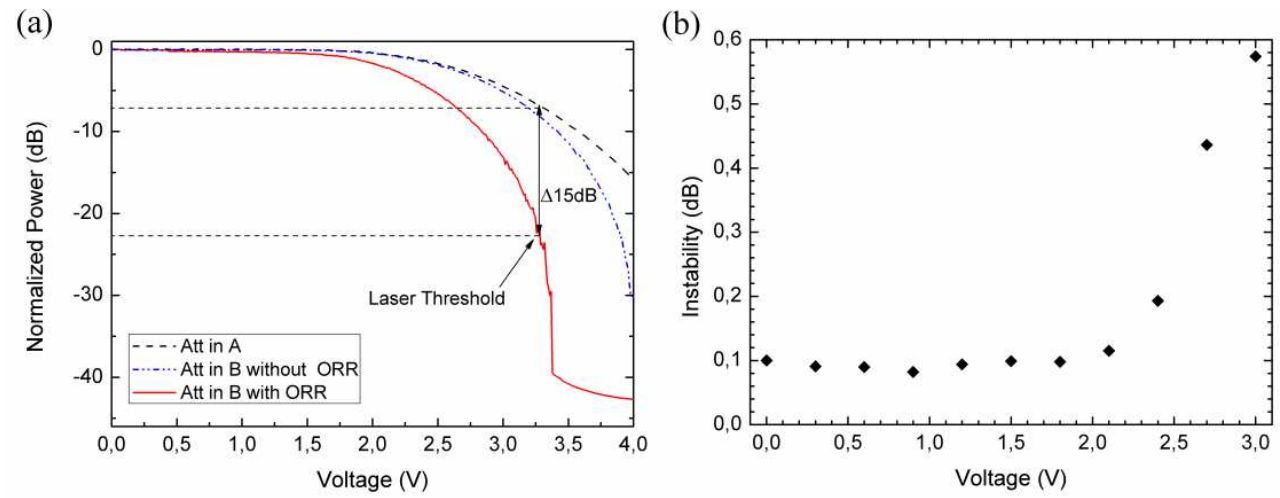

Figure 3. (a) Output power response against the voltage applied to VA located in position A (dash), position B (continuous) and position B without ORR (dash-dotted). (b) Output power instability of the structure against the voltage applied to VA.

The obtained results, figure $3 \mathrm{~b}$, show an instability close to $0.1 \mathrm{~dB}$ until the VA voltage reaches $2.1 \mathrm{~V}$. From this value onwards, the instability increases up to a maximum of $0.6 \mathrm{~dB}$, when a voltage of $3 \mathrm{~V}$ is applied to the attenuator. This is justified by the close operation of the system to the laser threshold.

In order to compare the instability with an external interrogation system, a characterization of the ORR with an external tunable laser source (TLS Agilent 81600B) was carried out. Figure 4a shows the experimental setup of the TLS interrogation method.

(a)

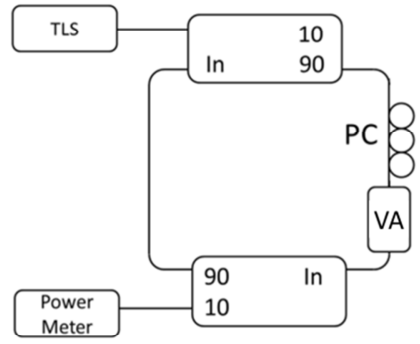

(b)

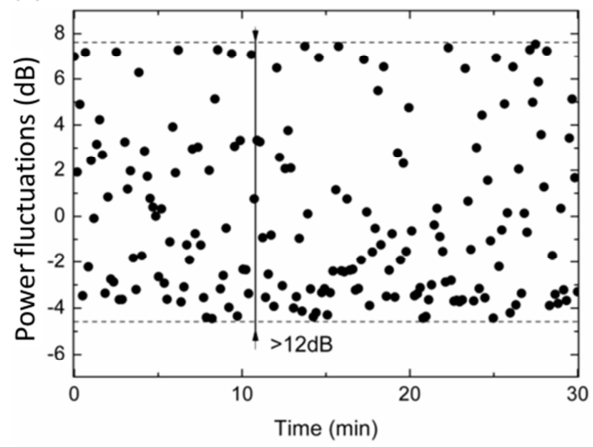

(c)

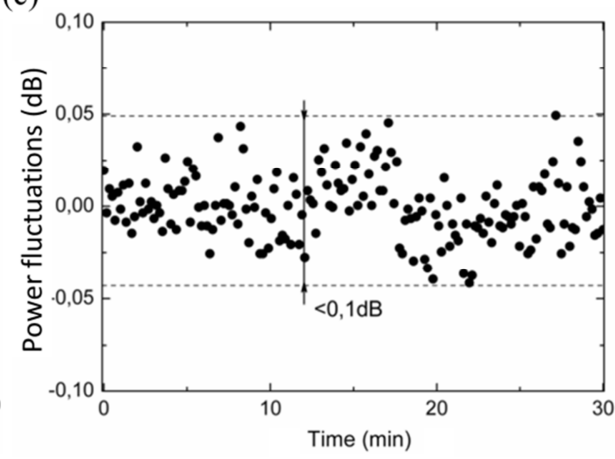

Figure 4. (a) Experimental setup of the TLS interrogation method. (b) Output power fluctuations of the TLS interrogation method (c) Output power fluctuations of the EDFRL interrogation method.

In figure $4 \mathrm{~b}$ and $4 \mathrm{c}$, it can be compared the instability without attenuation (VA=0dB) for the TLS interrogation method and the EDFRL sensor method, respectively, showing a remarkable instability improvement for the EDFRL interrogation. The problem of the external interrogation is due to the transfer function instability of the ring in combination with the TLS wavelength instability. The separation of peaks in the ORR transfer function is very narrow (around $95 \mathrm{MHz}$ ) so small TLS wavelength variations cause a substantial change of the detected output power.

\section{EDFRL SENSOR SYSTEM FOR DISPLACEMENT MEASUREMENT}

In this section a practical case for displacement measurements based on a bending sensor (Figure 5a) was investigated. The experimental setup for the EDFRL sensor system is the same as shown in figure 2 by replacing the V.A. by the bending sensor. Figure $5 \mathrm{~b}$ shows the measured results for the sensor at position $\mathrm{A}$ and $\mathrm{B}$. 
(a)

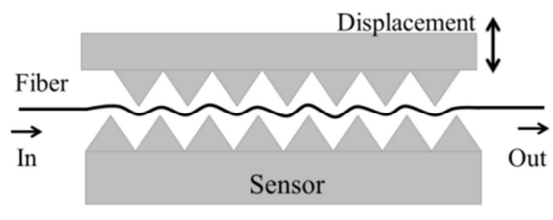

(b)

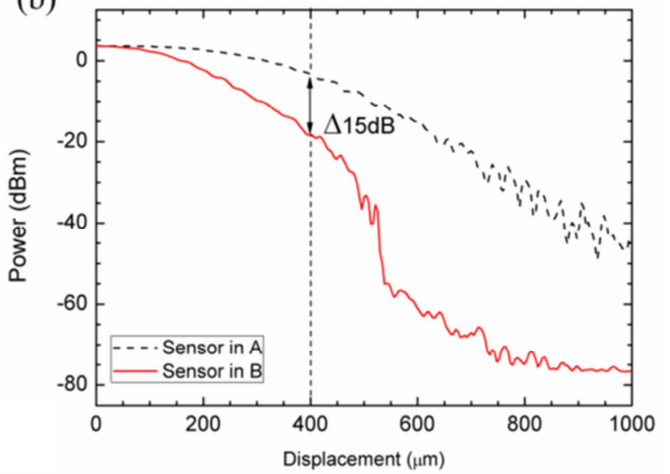

Fig. 5. (a) Schematic of the displacement sensor based on bending. (b) Output power response versus the displacement applied to the sensor, when located in position A (dotted) and B (continuous).

As seen above, when the sensor is located inside the ring, a dynamic range increment of $15 \mathrm{~dB}$ at $400 \mu \mathrm{m}$ was achieved. This configuration allows to measure up to $500 \mu \mathrm{m}$. An instability measure was also carried out at $400 \mu \mathrm{m}$, along 30 minutes, taking a sample each 10 seconds, showing a instability of $0.34 \mathrm{~dB}$

\section{CONCLUSIONS}

To summarize, in this paper a new system for enhancing fiber-optic intensity sensor measurements, based on a resonant ring cavity, is presented. The main advantage of this method is the ability to increase the dynamic range of an intensity sensor up to $15 \mathrm{~dB}$. In addition, because the reflected function of the ring-resonator is used to generate an EDFRL, the system works as a self-interrogator of the sensor and improve the instability in comparison with external interrogation methods. The proposed interrogation system is particularly suited for any kind of transmissive intensity sensors and only requires two active elements, an EDFA as gain medium and an optical power meter acting as detector. In addition, the system has been tested for a displacement sensor based on fiber optic bending showing an increment of the dynamic range of $15 \mathrm{~dB}$ at $400 \mu \mathrm{m}$ with a instability less than $0.34 \mathrm{~dB}$.

\section{ACKNOWLEDGMENTS}

The authors would like to acknowledge the financial support from the Spanish Ministerio de Educación y Ciencia through projects TEC2010-20224-C02-01, TEC2012-37958-C02-01 and Innocampus; from the European FEDER funding through ECOAL-MGT SUDOE project and from the European Research Council through Starting Grant UFINE (Grant No. 307441).

\section{REFERENCES}

[1] V. M. N. Passaro, C. de Tullio, B. Troia, M. La Notte, G. Giannoccaro, F. De Leonardis, "Recent Advances in Integrated Photonic Sensors," Sensors 12, 15558-15598,(2012).

[2] J. Saunders, M. A. Dreher, J. A. Barnes, C. M. Crudden, R. S. Brown, H. P. Loock, D. X. Xu, "Detection of volatile organic compounds with functionalized long-period gratings and micro-ring resonators," Proc. in 2011 ICO International Conference on Information Photonics, 1-2, (2011).

[3] C. Yu, Y. Zhang, X. Zhang, K. Wang, C. Yao, P. Yuan, Y. Guan, "Nested fiber ring resonator enhanced MachZehnder interferometer for temperature sensing," Appl. Opt. 51, 8873-8876, (2012).

[4] L. Zhang, P. Lu, L. Chen, C. Huang, D. Liu, S. Jiang, "Optical fiber strain sensor using fiber resonator based on frequency comb Vernier spectroscopy," Opt. Lett. 37, 2622-2624, (2012).

[5] Y. Guo and X. Fan, "Optofluidics in Bio-Chemical Analysis," Proc. SPIE, 8212, 82120F, (2012).

[6] M. Bravo, X. Angulo-Vinuesa, S. Martin-Lopez, M. Lopez-Amo, M. Gonzalez-Herraez, "Slow-light and enhanced sensitivity in a displacement sensor using a lossy fiber-based ring resonator," Journal of Light. Technol. 31, 37523757, (2013).

[7] C. Wang, "Fiber Loop Ringdown- a Time-Domain Sensing Thecnique for Multi-Function Fiber Otpic Sensor Platforms: Current Status and Design Perspectives," Sensors 9, pp. 7595-7621, (2009). 